International Journal of Pure and Applied Mathematics

Volume 99 No. 3 2015, 355-366

ISSN: 1311-8080 (printed version); ISSN: 1314-3395 (on-line version)

url: http://www.ijpam.eu

doi: http://dx.doi.org/10.12732/ijpam.v99i3.10

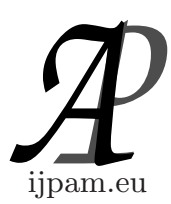

\title{
APPLICATION OF GENERALIZED DIFFERENTIAL TRANSFORM METHOD TO FRACTIONAL ORDER RICCATI DIFFERENTIAL EQUATION AND NUMERICAL RESULT
}

\author{
Manish Kumar Bansal ${ }^{1}$, Rashmi Jain ${ }^{2}$ \\ ${ }^{1,2}$ Department of Mathematics \\ Malaviya National Institute of Technology \\ Jaipur, 302017, INDIA
}

\begin{abstract}
In the present paper, we obtain the approximate solutions of Fractional Order Riccati Differential Equation by Generalized Differential Transform Method (GDTM). The fractional derivatives are described in the Caputo sense. Next we give eight numerical examples for sake of illustration of our method. Finally, we demonstrate our findings by means of two graphs by making use of MATHEMATICA software.
\end{abstract}

AMS Subject Classification: 26A33, 35A22, 65L05, 34A08

Key Words: Caputo fractional derivative, generalized differential transform method, fractional order Riccati differential equation, fractional differential equation

\section{Introduction}

The fractional calculus deals with derivatives and integrals to an arbitrary order (real or complex order). Fractional ordinary differential equations are crucial

Received: November 26, 2014

(C) 2015 Academic Publications, Ltd.

$\S_{\text {Correspondence author }}$ url: www.acadpubl.eu 
in many fields. For example, many physical phenomena in acoustics, damping laws and electroanalytical chemistry, neuron modeling, diffusion processing, and material sciences (see, e.g., [2]), [7] and [10] are described by differential equations of fractional order.

The general form of Fractional Order Riccati differential equation [15] is

$$
D^{\beta} y(t)=P(t) y^{2}(t)+Q(t) y(t)+R(t), \quad t>0
$$

Subject to initial condition

$$
y(0)=B
$$

where $\mathrm{P}(\mathrm{t}), \mathrm{Q}(\mathrm{t})$ and $\mathrm{R}(\mathrm{t})$ are known functions. For $\beta=1$, the fractionalorder Riccati differential equation converts into the classical Riccati differential equation. The use of fractional differential and integral operators in mathematical models has gained great importance in recent years. Several forms of fractional differential equations have been proposed in standard models, and there has been significant interest in developing numerical schemes for their solutions. There are numerous methods that deal with these types of equations; some of these methods are Laplace transform [20], Adomian decomposition method (ADM) [14], variational iteration method (VIM) [8], fractional difference method (FDM) [18], a quadrature tau method [4] and a shifted Jacobi spectral method [9]. Recently, there have been a number of schemes devoted to the solution of fractional differential equations. These schemes can be broadly classified into two classes, numerical and analytical.The homotopy perturbation method [19],[16],[3] and [1] homotopy analysis method [5] and [21] Taylor matrix method [13] and Haar wavelet method [17] have been used to solve the fractional-order Riccati differential equation. However, the convergence region of the corresponding results is rather small.

In the present paper we obtain analytic solution of Fractional-Order Riccati Differential Equation using generalized differential transform method [11]. The Differential Transform method was proposed by Zhou [22] to solve linear and nonlinear initial value problems in electric circuit analysis. This method constructs an analytical solution in the form of a series. It is different from the traditional higher order Taylor series method, which requires symbolic computation of the necessary derivatives of the data functions and takes long time in computation whereas the differential transform is an iterative procedure for obtaining analytic Taylor series solution. The method is further developed by Erturk, Momani and Odibat in their papers [11] for solving ordinary differential equations of fractional order. 


\section{Preliminaries}

\subsection{Definition}

The Caputo fractional derivative used in our present study is defined and represented in the following manner [6]

$$
D_{x}^{\alpha} f(x)=\frac{1}{\Gamma(m-\alpha)} \int_{x_{0}}^{x} \frac{f^{(m)}(\xi)}{(x-\xi)^{\alpha-m+1}} d \xi, \quad(m-1<\alpha \leq m), m \in N
$$

The Generalized Differential Transform. Consider the generalized differential transform $\left[11\right.$, p. 1647] of the $K^{\text {th }}$ derivative of function $\mathrm{f}(\mathrm{x})$ in one variable as follows:

$$
F_{\alpha}(k)=\frac{1}{\Gamma(\alpha k+1)}\left[\left(D_{x_{0}}^{\alpha}\right)^{k} f(x)\right]_{x=x_{0}}
$$

where $0<\alpha \leq 1,\left(D_{x_{0}}^{\alpha}\right)^{k}=D_{x_{0}}^{\alpha} \cdot D_{x_{0}}^{\alpha} \ldots D_{x_{0}}^{\alpha}(k-$ times $)$ and $F_{\alpha}(k)$ is the transformed function.

The Inverse Generalized Differential Transform of $F_{\alpha}(k)$ : (see [11, p. 1647])

$$
f(x)=\sum_{k=0}^{\infty} F_{\alpha}(k)\left(x-x_{0}\right)^{\alpha k}
$$

Some basic properties of the generalized differential transform given by equation (4) will also be required in the sequel:

Let $F_{\alpha}(k), G_{\alpha}(k)$ and $H_{\alpha}(k)$ be the generalized differential transforms of the functions $\mathrm{f}(\mathrm{x}), \mathrm{g}(\mathrm{x})$ and $\mathrm{h}(\mathrm{x})$ respectively, then:

(a) If $\mathrm{f}(\mathrm{x})=\mathrm{g}(\mathrm{x}) \pm \mathrm{h}(\mathrm{x})$, then $F_{\alpha}(k)=G_{\alpha}(k) \pm H_{\alpha}(k)$

(b) If $\mathrm{f}(\mathrm{x})=\mathrm{ag}(\mathrm{x})$, then $F_{\alpha}(k)=a G_{\alpha}(k)$, where a is a constant.

(c) If $\mathrm{f}(\mathrm{x})=\mathrm{g}(\mathrm{x}) \mathrm{h}(\mathrm{x})$, then $F_{\alpha}(k)=\sum_{l=0}^{k} G_{\alpha}(l) H_{\alpha}(k-l)$

(d) If $\mathrm{f}(\mathrm{x})=D_{x_{0}}^{\alpha} g(x)$, then $F_{\alpha}(k)=\frac{\Gamma(\alpha(k+1)+1)}{\Gamma(\alpha k+1)} G_{\alpha}(k+1)$

(e) If $\mathrm{f}(\mathrm{x})=\left(x-x_{0}\right)^{\gamma}, \gamma=n \alpha, n \in Z$, then $F_{\alpha}(k)=\delta(k-\gamma / \alpha)$, where

$$
\delta(k)=\left\{\begin{array}{cc}
1, & \text { if } \quad k=0 \\
0, & \text { otherwise }
\end{array}\right.
$$


(f) If $f(x)=D_{x_{0}}^{\beta} g(x), m-1<\beta \leq m$ and the function $\mathrm{g}(\mathrm{x})$ satisfies the condition in Theorem 2 , then

$$
F_{\alpha}(k)=\frac{\Gamma(\alpha k+\beta+1)}{\Gamma(\alpha k+1)} G_{\alpha}(k+\beta / \alpha)
$$

\subsection{Known Basic Theorems}

The following theorems will be required to obtain our main findings:

Theorem 1. (Generalized Taylor Formula) Suppose that $\left(D_{a}^{\alpha}\right)^{k} f(x) \in$ $C[a, b]$ for $k=0,1,2, \ldots, n+1$, where $0<\alpha \leq 1$, then we have

$$
f(x)=\sum_{i=o}^{n} \frac{(x-a)^{i \alpha}}{\Gamma(i \alpha+1)}\left(\left(D_{a}^{\alpha}\right)^{i} f\right)(a)+\frac{\left(\left(D_{a}^{\alpha}\right)^{n+1} f\right)(\xi)}{\Gamma((n+1) \alpha+1)} \cdot(x-a)^{(n+1) \alpha}
$$

with $a \leq \xi \leq x, \forall \quad x \in[a, b]$. The above theorem was recently obtained by Erturk et al.[11, p. 1645].

Theorem 2. Suppose that $f(x)=\left(x-x_{0}\right)^{\lambda} g(x)$, where $\lambda>0$ and $g(x)$ has the generalized power series expansion $g(x)=\sum_{n=0}^{\infty} a_{n}\left(x-x_{0}\right)^{n \alpha}$ with radius of convergence $R>0,0<\alpha \leq 1$. Then

$$
D_{a}^{\gamma} D_{a}^{\beta} f(x)=D_{a}^{\gamma+\beta} f(x)
$$

for all $\left(x-x_{0}\right) \in(0, R)$, the coefficients $a_{n}=0$ for $n$ gives by $n \alpha-\lambda+\beta=0$ and either $\lambda>\mu, \mu=\max (\beta+[\gamma],[\beta+\gamma])$

or

$\lambda \leq \mu, a_{k}=0$ for $k=0,1,2, \ldots,\left[\frac{\mu-\lambda}{\alpha}\right]$

where $[\mathrm{x}]$ denotes the greatest integer less than or equal to $x$.

The above theorem was recently obtained by Garg et al. [12].

\section{Solution of Fractional Order Riccati Differential Equation by GDTM}

Consider the Fractional Order Riccati Differential Equation:

$$
D^{\beta} y(t)=P(t) y^{2}(t)+Q(t) y(t)+R(t), \quad t>0, \quad 0<\beta \leq 1
$$


Subject to initial condition

$$
y(0)=B
$$

where $\mathrm{P}(\mathrm{t}), \mathrm{Q}(\mathrm{t})$ and $\mathrm{R}(\mathrm{t})$ are known functions and $\left(D_{a}^{\alpha}\right)^{k} y(t) \in C[a, b]$ for $\mathrm{k}$ $=0,1,2, \ldots, \mathrm{n}+1,0<\alpha \leq 1$ given in Theorem 1. Taking the Generalized Differential Transform defined by (4) on both sides of equation (9) and making use of its property (6), we easily arrive at the following result after a little simplification

$$
\begin{aligned}
& \begin{array}{l}
\frac{\Gamma(\alpha k+\beta+1)}{\Gamma(\alpha k+1)} Y_{\alpha}\left(k+\frac{\beta}{\alpha}\right)=\sum_{k=0}^{h} \sum_{l=0}^{k} P_{\alpha}(l) Y_{\alpha}(k-l) Y_{\alpha}(h-k) \\
+\sum_{l=0}^{k} Q_{\alpha}(l) Y_{\alpha}(k-l)+R_{\alpha}(k)
\end{array} \\
& \Rightarrow Y_{\alpha}\left(k+\frac{\beta}{\alpha}\right)= \\
& \frac{\Gamma(\alpha k+1)}{\Gamma(\alpha k+\beta+1)}\left[\sum_{k=0}^{h} \sum_{l=0}^{k} P_{\alpha}(l) Y_{\alpha}(k-l) Y_{\alpha}(h-k)+\sum_{l=0}^{k} Q_{\alpha}(l) Y_{\alpha}(k-l)+R_{\alpha}(k)\right]
\end{aligned}
$$

where $Y_{\alpha}(k)$ is the Generalized Differential Transform function of $\mathrm{y}(\mathrm{t})$.

Using the initial condition given by (10), the operator represented by (4) takes the form

$$
Y_{\alpha}(k)=\frac{1}{\Gamma(\alpha k+1)}\left[\left(D_{t_{0}}^{\alpha}\right)^{k} y(t)\right]_{t=0} \quad \text { for } \quad k=0,1,2, \ldots,
$$

taking the inverse generalized differential transform to equation (12), we obtain the desired solution with the help of eq.(13) after a little simplification.

\subsection{Examples}

In order to illustrate the effectiveness of the method proposed, several numerical examples are given in this section.

Example 1. If we take $\beta=\frac{1}{4}, \mathrm{P}(\mathrm{t})=1, \mathrm{Q}(\mathrm{t})=2$ and $\mathrm{R}(\mathrm{t})=t^{2}$ in equation (9), it reduces to

$$
D^{\frac{1}{4}} y(t)=t^{2}+2 y(t)+y^{2}(t)
$$


Subject to initial condition

$$
y(0)=0
$$

on taking generalized differential transform (4) with $t_{0}=0$ and setting $\alpha=1 / 4$ in equation (12) with condition (13), to both sides of equation (14) and making use of property (6) of generalized differential transform, equation (14) takes the following form

$$
Y_{\frac{1}{4}}(k+1)=\frac{\Gamma\left(\frac{k}{4}+1\right)}{\Gamma\left(\frac{k}{4}+\frac{5}{4}\right)}\left[\delta(k-8)+2 Y_{\frac{1}{4}}(k)+\sum_{l=0}^{k} Y_{\frac{1}{4}}(l) Y_{\frac{1}{4}}(k-l)\right]
$$

where

$$
Y_{\frac{1}{4}}(0)=0
$$

Now making use of the recurrence relation (16) and the initial condition (17), for $\mathrm{k}=0,1,2, \ldots$ we obtain after a little simplification the following values of $Y_{\frac{1}{4}}(k)$ for $\mathrm{k}=1,2,3 \ldots$,

$$
\begin{gathered}
Y_{\frac{1}{4}}(1)=0 ; \quad Y_{\frac{1}{4}}(2)=0 ; \quad Y_{\frac{1}{4}}(3)=0 ; \quad Y_{\frac{1}{4}}(4)=0 ; \quad Y_{\frac{1}{4}}(5)=0 \\
Y_{\frac{1}{4}}(6)=0 ; \quad Y_{\frac{1}{4}}(7)=0 ; \quad Y_{\frac{1}{4}}(8)=0 ; \quad Y_{\frac{1}{4}}(9)=0.6018 ; \quad Y_{\frac{1}{4}}(10)=1.204 \\
Y_{\frac{1}{4}}(11)=1.809 ; \quad Y_{\frac{1}{4}}(12)=2.667 ; \quad Y_{\frac{1}{4}}(13)=3.862 ; \quad Y_{\frac{1}{4}}(14)=5.502 \\
Y_{\frac{1}{4}}(15)=7.717 ; \quad Y_{\frac{1}{4}}(16)=10.67
\end{gathered}
$$

Similarly we can find the value of $Y_{\frac{1}{4}}(k)$ for $\mathrm{k}=17,18,19 \ldots$

Again if in the inverse transform given by equation (5), we take $\alpha=\frac{1}{4}, t_{0}=0$ and $\mathrm{f}(\mathrm{t})$ is replaced by $\mathrm{y}(\mathrm{t})$ then it takes the following form

$$
y(t)=\sum_{k=0}^{\infty} Y_{\frac{1}{4}}(k) t^{\frac{k}{4}}
$$

Using the values of $Y_{\frac{1}{4}}(k)$ From equation (18) in equation (19), a solution of Fractional Order Reccati Differential Equation (14) obtained as

$$
\begin{aligned}
y(t)=0.6018 t^{\frac{9}{4}}+1.204 t^{\frac{10}{4}}+1.809 t^{\frac{11}{4}}+ & 2.667 t^{3}+3.862 t^{\frac{13}{4}} \\
& +5.502 t^{\frac{14}{4}}+7.717 t^{\frac{15}{4}}+10.67 t^{4} \ldots .
\end{aligned}
$$


Example 2. If we take $\beta=\frac{1}{2}, \mathrm{P}(\mathrm{t})=1, \mathrm{Q}(\mathrm{t})=2$ and $\mathrm{R}(\mathrm{t})=t^{2}$ in equation (9) and follow the method given in Example 1, we easily arrive at the following result

$$
\begin{aligned}
y(t)=0.6018 t^{\frac{5}{2}}+0.6667 t^{3}+0.6878 t^{\frac{7}{2}}+0.6667 t^{4}+0.6114 t^{\frac{9}{2}} \\
+0.5334 t^{5}+0.6599 t^{\frac{11}{2}}+0.8485 t^{6} \ldots
\end{aligned}
$$

Example 3.If we take $\beta=\frac{3}{4}, \mathrm{P}(\mathrm{t})=1, \mathrm{Q}(\mathrm{t})=2$ and $\mathrm{R}(\mathrm{t})=t^{2}$ in equation (9) and follow the method given in Example 1, we easily arrive at the following result

$$
y(t)=0.4522 t^{\frac{11}{4}}+0.3439 t^{\frac{14}{4}}+0.2272 t^{\frac{17}{4}}+0.133 t^{5} \ldots
$$

Example 4. If we take $\beta=1, \mathrm{P}(\mathrm{t})=-1, \mathrm{Q}(\mathrm{t})=2$ and $\mathrm{R}(\mathrm{t})=t^{2}$ in equation (9) and follow the method given in Example 1, we easily arrive at the following result

$$
y(t)=\frac{t^{3}}{3}+\frac{t^{4}}{6}+\frac{t^{5}}{15}+\frac{t^{6}}{45}+\frac{t^{7}}{45} \ldots
$$

The graph given below demonstrates have been represented eq.(20) to eq. (23) graphically by making use of MATHEMATICA software as given in Figure 1.

Example 5. If we take $\beta=\frac{1}{4}, \mathrm{P}(\mathrm{t})=-1, \mathrm{Q}(\mathrm{t})=3$ and $\mathrm{R}(\mathrm{t})=\mathrm{t}$ in equation (9), it reduces to

$$
D^{\frac{1}{4}} y(t)=t+3 y(t)-y^{2}(t)
$$

Subject to initial condition

$$
y(0)=1
$$

on taking generalized differential transform (4) with $t_{0}=0$ and setting $\alpha=1 / 4$ in equation (12) with condition (13), to both sides of equation (24) and making use of property (6)of generalized differential transform, equation (24) takes the following form

$$
Y_{\frac{1}{4}}(k+1)=\frac{\Gamma\left(\frac{k}{4}+1\right)}{\Gamma\left(\frac{k}{4}+\frac{5}{4}\right)}\left[\delta(k-4)+3 Y_{\frac{1}{4}}(k)-\sum_{l=0}^{k} Y_{\frac{1}{4}}(l) Y_{\frac{1}{4}}(k-l)\right]
$$

where

$$
Y_{\frac{1}{4}}(0)=1
$$

Now making use of the recurrence relation (26) and the initial condition (27), for $\mathrm{k}=0,1,2, \ldots$ we obtain after a little simplification the following value of $Y_{\frac{1}{4}}(k)$ for $\mathrm{k}=1,2,3 \ldots$, 


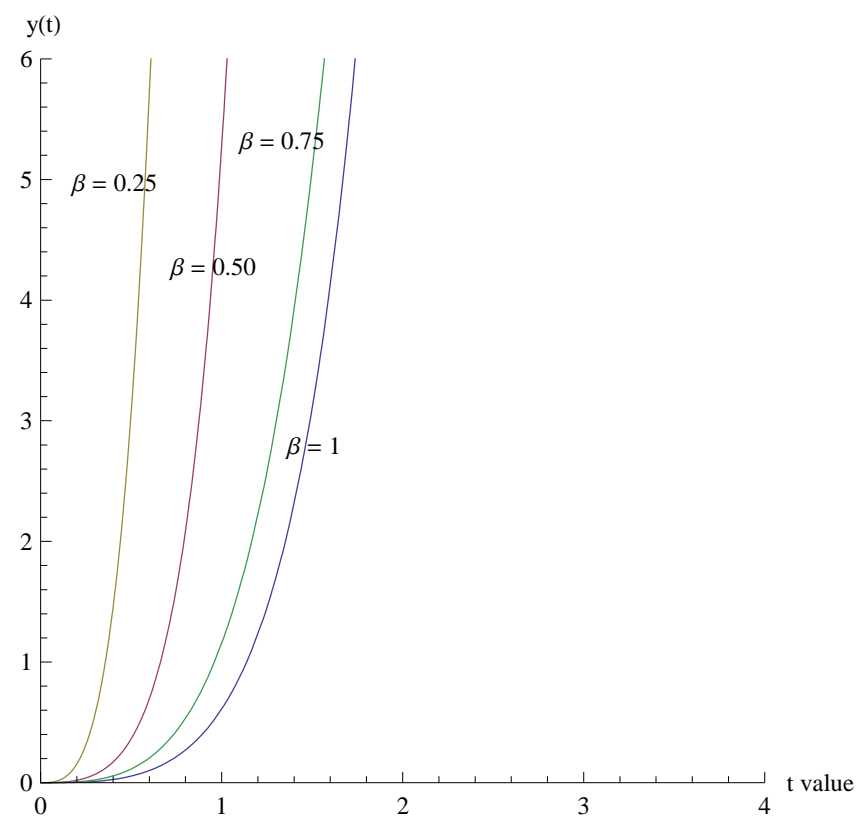

Figure 1: Approxiamte solution for Example 1

$$
\begin{aligned}
& Y_{\frac{1}{4}}(1)=2.207 ; \quad Y_{\frac{1}{4}}(2)=2.2578 ; \quad Y_{\frac{1}{4}}(3)=-2.519 \\
& Y_{\frac{1}{4}}(4)=-11.4756 ; \quad Y_{\frac{1}{4}}(5)=-3.9283 ; \quad Y_{\frac{1}{4}}(6)=49.5215
\end{aligned}
$$

Similarly we can find the value of $Y_{\frac{1}{4}}(k)$ for $\mathrm{k}=7,8,9 \ldots$.

Again if in the inverse transform given by equation (5), we take $\alpha=\frac{1}{4}, t_{0}=0$ and $f(t)$ is replaced by $y(t)$ then it takes the following form

$$
y(t)=\sum_{k=0}^{\infty} Y_{\frac{1}{4}}(k) t^{\frac{k}{4}}
$$

Using the values of $Y_{\frac{1}{4}}(k)$ From equation (28) in equation (29), a solution of Fractional Order Reccati Differential Equation (24) obtained as

$$
y(t)=1+2.207 t^{\frac{1}{2}}+2.2578 t-2.5198 t^{\frac{3}{2}}-11.4756 t^{2}-3.9283 t^{\frac{5}{2}}+49.5215 t^{3} \ldots
$$

Example 6/ If we take $\beta=\frac{1}{2}, \mathrm{P}(\mathrm{t})=-1, \mathrm{Q}(\mathrm{t})=3$ and $\mathrm{R}(\mathrm{t})=\mathrm{t}$ in equation (9) and follow the method given in Example 5, we easily arrive at the following result

$$
y(t)=1+2.257 t^{\frac{1}{2}}+2 t-1.5753 t^{\frac{3}{2}}-7.048 t^{2}-2.3694 t^{\frac{5}{2}}+19.7999 t^{3} \ldots
$$




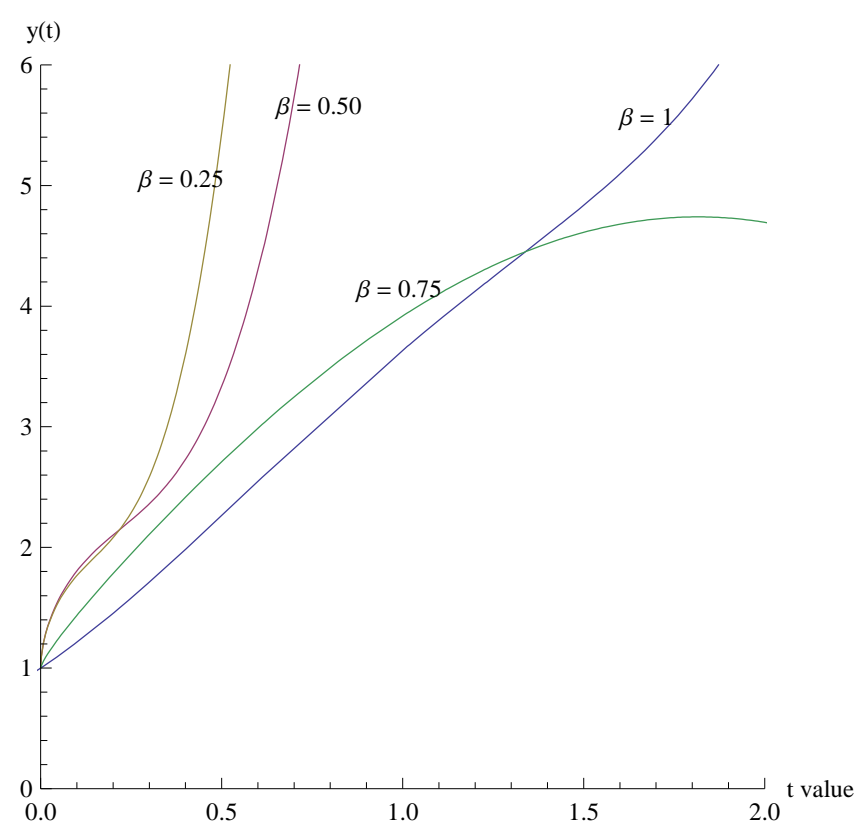

Figure 2: Approxiamte solution for Example 2

Example 7. If we take $\beta=\frac{3}{4}, \mathrm{P}(\mathrm{t})=-1, \mathrm{Q}(\mathrm{t})=3$ and $\mathrm{R}(\mathrm{t})=\mathrm{t}$ in equation (9) and follow the method given in Example 5, we easily arrive at the following result

$$
y(t)=1+2.176 t^{\frac{3}{4}}+1.5045 t^{\frac{6}{4}}-1.6847 t^{\frac{9}{4}}+0.3009 t^{\frac{10}{4}} \ldots
$$

Example 8: If we take $\beta=1, \mathrm{P}(\mathrm{t})=-1, \mathrm{Q}(\mathrm{t})=3$ and $\mathrm{R}(\mathrm{t})=\mathrm{t}$ in equation (9) and follow the method given in Example 5, we easily arrive at the following result

$$
y(t)=1+2 t+1.5 t^{2}-0.8333 t^{3}-0.2083 t^{4}+0.1749 t^{5} \ldots
$$

The graph given below demonstrates have been represented eq.(30) to eq. (33) graphically by making use of MATHEMATICA software as given in Figure 2 .

\section{Conclusion}

In the present study, we have applied the Generalized Differential Transform Method (GDTM) to find the approximate solution of Fractional Order Riccati differential Equation. We have also given the graphical representation of our findings. 


\section{References}

[1] Abbasbandy, S. (2006). Iterated Hes homotopy perturbation method for quadratic Riccati differential equation. Appl. Math. Comput., 175, 581589.

[2] Al-Mdallal,Q. M. , Syam,M. I. and Anwar,M. N.(2010). A collocationshooting method for solving fractional boundary value problems.Communications in Nonlinear Science and Numerical Simulation,15(12), 3814-3822.

[3] Aminkhah, H. and Hemmatnezhad, M. (2010). An efficient method for quadratic Riccati differential equation.Commun. Nonlinear Sci. Numer. Simul.,15, 835-839.

[4] Bhrawy,A. H., Alofi,A. S. and Ezz-Eldien,S. S. (2011). A quadrature tau method for fractional differential equations with variable coefficients. $A p$ plied Mathematics Letters, 24(12), 2146-2152.

[5] Cang, J., Tan, Y., Xu, H. and Liao, S. J. (2009). Series solutions of nonlinear Riccati differential equations with fractional order. Chaos Solitons Fractals, 40, 1-9.

[6] Caputo,M.(1967). Linear models of dissipation whose Q is almost frequency independent-II. Geophysical Journal International,13(5),529-539.

[7] Daftardar-Gejji, V. and Jafari,H. (2005). Adomian decomposition: a tool for solving a system of fractional differential equations. Journal of Mathematical Analysis and Applications,301(2), 508-518.

[8] Das,S. (2009). Analytical solution of a fractional diffusion equation by variational iteration method. Computers and Mathematics with Applications, 57(3), 483-487.

[9] Doha,E.H., Bhrawy,A. H., Baleanu,D. and Ezz-Eldien,S. S. (2013). On shifted Jacobi spectral approximations for solving fractional differential equations. Applied Mathematics and Computation, 219(15), 8042-8056.

[10] Enesiz,Y. C, Keskin,Y. and Kurnaz,A. (2010). The solution of the BagleyTorvik equation with the generalized Taylor collocation method. Journal of the Franklin Institute Engineering and Applied Mathematics,347(2), 452-466. 
[11] Erturk,V.S.,Momani,S. and Odibat,Z.(2008). Application of generalized differential transform method to multi-order fractional differential equations.Communications in Nonlinear Science and Numerical Simulation,13(8), 1642-1654.

[12] Garg,M. and Manohar,P.(2015). Three-dimensional generalized differential transform method for space-time fractional diffusion equation, Palestine Journal of Mathematics,4(1),127-135.

[13] Gülsu, M. and Sezer, M. (2006). On the solution of the Riccati equation by the Taylor matrix method. Appl. Math. Comput., 176(2), 414-421.

[14] Jafari, H. and Seifi,S.(2009). Solving a system of nonlinear fractional partial differential equations using homotopy analysis method. Communications in Nonlinear Science and Numerical Simulation, 14(5), 1962-1969.

[15] Khan, N.A., Ara, A. and Khan, N.A. (2013). Fractional-order Riccati differential equation: Analytical approximation and numerical results. Advances in Difference Equations, 2013, 1-16.

[16] Khan, N.A., Ara, A. and Jamil, M. (2011). An efficient approach for solving the Riccati equation with fractional orders. Comput. Math. Appl, 61, 26832689 .

[17] Li, Y. and Hu, L. (2010). Solving fractional Riccati differential equations. Third International Conference on Information and Computing Using Haar Wavelet. IEEE

[18] Meerschaert, M.M. and Tadjeran,C. (2006). Finite difference approximations for two-sided space-fractional partial differential equations. Applied Numerical Mathematics: An IMACS Journal, 56(1), 80-90.

[19] Odibat, Z., Momani, S. (2008). Modified homotopy perturbation method: application to quadratic Riccati differential equation of fractional order. Chaos Solitons Fractals, 36(1), 167-174.

[20] Podlubny,I.(1999).Fractional Differential Equations.California,USA: Academic Press.

[21] Tan, Y. and Abbasbandy, S. (2008). Homotopy analysis method for quadratic Riccati differential equation. Commun. Nonlinear Sci. Numer. Simul., 13, 539-546. 
[22] Zhou, JK.(1986).Differential transformation and its applications for electrical circuits. Wuhan, China: Huazhong University Press. 